Int. J. Pharm. Regular Article

\title{
Hybrid vector including polyethylenimine and cationic lipid, DOTMA, for gene delivery
}

Megumi Matsumoto, Reiko Kishikawa, Tomoaki Kurosaki, Hiroo Nakagawa, Nobuhiro Ichikawa, Tomoyuki Hamamoto, Hideto To, Takashi Kitahara, Hitoshi Sasaki ${ }^{*}$

Department of Hospital Pharmacy, Nagasaki University School of Medicine, 1-7-1 Sakamoto, Nagasaki 852-8501, Japan.

*Correspondence: Department of Hospital Pharmacy, Nagasaki University School of Medicine, 1-7-1 Sakamoto, Nagasaki 852-8501, Japan.

Fax: $\quad+81-95-819-7251$

Tel.: $\quad+81-95-819-7245$

e-mail: $\quad$ sasaki@net.nagasaki-u.ac.jp 


\section{Abstract}

We developed polyethylenimine (PEI) lipopolyplexes with N-[1-(2, 3-dioleyloxy) propyl]-N, N, N-trimethlylammonium chloride (DOTMA) and pDNA to investigate their usefulness for in vitro and in vivo gene delivery. The charge ratio of the complex to pDNA was calculated with molar values of nitrogen of PEI, and nitrogen of DOTMA to phosphate of pDNA. The polyplexes were prepared at charge ratio 2 (polyplex $2 \mathrm{P}$ ) and 8 (polyplex $8 \mathrm{P}$ ). DOTMA solution was added to polyplex 2P to prepare lipopolyplexes at charge ratio 1 (lipopolyplex 2P-1D), 2 (lipopolyplex 2P-2D), and 4 (lipopolyplex 2P-4D). The particle size of the complex was significantly reduced by the addition of DOTMA and settled to 74-114 nm, indicating pDNA compaction. The addition of DOTMA to polyplex 2P decreased pDNA dissociation from the complex and degradation in serum. The addition of DOTMA to polyplex 2P remarkably increased gene expression in HepG2 cells in the absence or presence of FBS. These lipopolyplexes showed little cytotoxicity in the presence of FBS. After intravenous injection of the lipopolyplexes into mice, high gene expression in the liver, spleen, and lung was observed with lipopolyplex 2P-2D, lipopolyplex 2P-4D, and polyplex 8P. In particular, lipopolyplex 2P-4D showed the highest gene expression.

KEY WORDS: gene delivery; hybrid vector; non-viral vector; luciferase; polyethylenimine; cationic lipid 


\section{Introduction}

Gene therapy is expected to be an effective method to treat cancer, infection, innate immunodeficiency, and cardiovascular diseases (Verma et al., 1997). The success of gene therapy highly depends on the development of effective and secure delivery vectors. The gene delivery vector is categorized into viral and non-viral vectors. The viral vector is highly effective and has been used in clinical trials, although some severe adverse events were of great concern for its safety (Edelstein et al., 2007). On the other hand, non-viral vectors have safety advantages: much lower immunotoxicity, a clear structure, and easy modeling; therefore, much attention has been paid to non-viral vectors (Tang et al., 1997; Fillion et al., 1998).

The conformation of the pDNA-vector complex is an important factor that influences transgene efficiency. Two major forms of non-viral vectors are polyplex (complex of pDNA and cationic polymer) and lipoplex (complex of pDNA and cationic liposomes). These are independently effective for gene delivery in several cell lines and in vivo experiments (Gao et al., 1995; Goldman et al., 1997; Sorgi et al., 1997). Recently, a ternary complex of cationic polymer, cationic liposomes, and pDNA, named lipopolyplex, has been developed as a second-generation non-viral vector. Lipopolyplexes have been reported to show extremely high gene expression by synergism of cationic polymers and liposomes (Li et al., 1997; Lampela et al., 2003; Lee et al., 2003).

Polyethylenimine (PEI) is a popular cationic polymer for polyplex and has shown high gene expression in in vitro and in vivo gene delivery studies (Godbey et al., 1999; Kircheis et al., 2001). Several advantages of PEI in the process of gene transfection have been reported; condensing pDNA by electrostatic interaction, binding to the cell surface and taken up by the endocytotic pathway, and releasing pDNA into the cytoplasm, via the so-called 'proton sponge mechanism' (Boussif et al., 1995; Kichler et al., 2001; Itaka et al., 2004). It was also able to accelerate gene entry into the nucleus from the cytoplasm (Pollard et al., 1998). Furthermore, lipopolyplexes of PEI with DOCSPER, DOSPER, and DOTAP demonstrated high gene expression in in vitro experiments 
(Lampela et al., 2003; Pelisek et al, 2006); however, there have been few reports about the usefulness of PEI lipopolyplex in in vivo experiments. In the preliminary experiment, we found that a lipopolyplex of PEI with $\mathrm{N}$-[1-(2, 3-dioleyloxy) propyl]-N, N, N-trimethlylammonium chloride (DOTMA) showed higher gene expression than polyplex of PEI in in vivo experiments; therefore, we investigated the usefulness of PEI lipopolyplex with DOTMA and pDNA in in vitro and in vivo gene delivery. 


\section{Materials and methods}

\subsection{Chemicals}

PEI (branched form, average molecular weight of 25,000) was purchased from Aldrich Chemical Co. (Milwaukee, WI, USA). DOTMA was purchased from Tokyo Chemical Industry Co. Ltd. (Tokyo, Japan). Fetal bovine serum (FBS) was obtained from Biological Industries Ltd. (Kibbutz Beit Haemek, Israel). Bovine serum albumin (BSA, minimum 98\%, lyophilized powder and low endotoxin) was obtained from Sigma Chemical Co. (St. Louis, MO, USA). Dulbecco's modified Eagle's medium (DMEM), antibiotics (penicillin $100 \mathrm{U} / \mathrm{mL}$; streptomycin $100 \mu \mathrm{g} / \mathrm{mL}$ ) and other culture reagents were obtained from Invitrogen Corp. (Carlsbad, CA, USA). Phosphate-buffered saline (PBS) was prepared by mixing isotonic phosphate buffer ( $\mathrm{pH}$ 7.4) with an equal volume of saline. WST-1 (2-(4-lodophenyl)-3-(4-nitrophenyl)-2H-tetrazolium, monosodium salt) and 1-methoxy PMS (1-methoxy-5-methylphenazinium methylsulfate) were obtained from Dojindo Laboratories (Kumamoto, Japan). Lissamine rhodamine B 1, 2-dihexadecanoylsn-glycero-3-phosphoethanolamine, triethylammonium salt (Rh-DHPE) and Hoechst 33342 were purchased from Molecular Probes (Leiden, The Netherlands). Rhodamine-PEI (Rh-PEI) was prepared in our laboratory. Briefly, PEI and rhodamine B isothiocyanate (Aldrich Chemical Co., Milwaukee, WI, USA) were dissolved in dimethyl sulfoxide (DMSO) and stirred overnight at room temperature in the dark. Rh-PEI was purified by gel filtration. Almost $1.5 \%$ of PEI nitrogen was labeled with rhodamine B. All other chemicals were of reagent grade and used as obtained commercially.

\subsection{Construction of $p D N A$}

pCMV-luciferase was constructed by subcloning the HindIII/Xbal firefly luciferase cDNA fragment from the pGL3-control vector (Promega, Madison, WI, U.S.A.) into the polylinker of the pcDNA3 vector (Invitrogen, Carlsbad, CA, U.S.A.). Green fluorescence protein (GFP) encoding the 
plasmid (pEGFP-C1) was purchased from Clontech (Palo Alto, CA, USA). pDNA was amplified using a EndoFree ${ }^{\circledR}$ Plasmid Giga Kit (QIAGEN GmbH, Hilden, Germany). pDNA was dissolved in $5 \%$ dextrose solution and stored at $-80^{\circ} \mathrm{C}$ until analysis. The pDNA concentration was measured at absorbance of $260 \mathrm{~nm}$ and adjusted to $1 \mathrm{mg} / \mathrm{ml}$.

\subsection{Preparation of polyplex and lipopolyplex}

The theoretical charge ratio of pDNA/PEI complexes was calculated as the molar ratio of nitrogen of PEI to phosphate of DNA. An appropriate amount of stock PEI solution (pH 7.4) was mixed with the diluted solution of pDNA $(1 \mathrm{mg} / \mathrm{ml})$ by pipetting thoroughly to prepare polyplexes at charge ratio 2 (polyplex 2P) and 8 (polyplex 8P). For preparation of lipopolyplexes, the theoretical charge ratio of DOTMA to pDNA was calculated as the molar ratio of the nitrogen of DOTMA to the phosphate of pDNA. DOTMA ethanol solution was added to polyplex 2P after 10 minutes, and left for a further 20 minutes at room temperature to prepare lipopolyplexes at a charge ratio of DOTMA/pDNA 1 (lipopolyplex 2P-1D), 2 (lipopolyplex 2P-2D), and 4 (lipopolyplex 2P-4D).

\subsection{Evaluation of physicochemical properties of lipopolyplexes}

The particle size and zeta potential of polyplexes and lipopolyplexes were measured with Zetasizer Nano ZS (Malvern Instruments, Ltd., United Kingdom). The number-fractioned mean diameter is shown. Transmission electron microscopy (JEM-1210; JEOL, Tokyo, Japan) was also used to evaluate the particle size of complexes.

\subsection{Electrophoresis assay}

Five microliter aliquots of complex solution were mixed with $1 \mu$ l of loading buffer ( $30 \%$ glycerol and $0.2 \%$ bromophenol blue) and loaded onto a $0.6 \%$ agarose gel. Electrophoresis (i-Mupid $\mathrm{J}^{\mathbb{B}}$; Cosmo Bio, Tokyo, Japan) was carried out at $50 \mathrm{~V}$ in running buffer solution (40 mM Tris/ $\mathrm{HCl}, 40$ 
$\mathrm{mM}$ acetic acid and $1 \mathrm{mM}$ EDTA). The retardation of complexes was visualized with ethidium bromide staining.

\subsection{In vitro gene expression experiments}

The human hepatoma cell line HepG2 was obtained from the Cell Resource Center for Biomedical Research, Tohoku University. HepG2 cells were maintained in DMEM supplemented with $10 \%$ FBS and antibiotics under a humidified atmosphere of $5 \% \mathrm{CO}_{2}$ in air at $37^{\circ} \mathrm{C}$. The cells were plated on 12-well collagen-containing plates (Becton-Dickinson, Franklin Lakes, NL, USA.) at a density $5.0 \mathrm{x}$ $10^{4}$ cells/well and cultivated in $1.5 \mathrm{ml}$ DMEM supplemented with $10 \%$ FBS and antibiotics. In the transfection experiment for complexes, after $24 \mathrm{~h}$ preincubation, the medium was replaced with 1.5 ml DMEM with or without FBS. Two $\mu$ g of pDNA complexed with PEI and DOTMA was then added to each well and incubated for $2 \mathrm{~h}$. After transfection, the incubation medium was removed and cells were cultured for a further $22 \mathrm{~h}$ at $37^{\circ} \mathrm{C}$ with DMEM supplemented with $10 \%$ FBS and antibiotics until luciferase analysis. For the evaluation of GFP expression, HepG2 cells were seeded at a density of $1.0 \times 10^{5}$ cells/well and transfected with GFP encoding pDNA (pEGFP) polyplexes and lipopolyplexes in the presence of FBS, as described above. The relative levels of GFP expression were characterized using fluorescent microscopy $24 \mathrm{~h}$ after transfection.

\subsection{Evaluation of intracellular distribution of lipopolyplex}

To observe the intracellular distribution of lipopolyplexes, HepG2 cells were transfected as described above with pEGFP complexed with PEI and DOTMA, including fluorescent dye such as Rh-PEI or Rh-DHPE. At $24 \mathrm{~h}$ after transfection, cells were incubated with Hoechst 33342 containing culture medium for $30 \mathrm{~min}$ to visualize nuclei and then medium was replaced, and fluorescence distribution of Rh-PEI, Rh-DHPE, Hoechst 33342, and GFP was observed with fluorescent microscopy. The tone of each image was adjusted and overlapped to give a merged picture by digital 
processing.

\subsection{In vivo gene expression experiments}

Animal care and experimental procedures were performed in accordance with the Guidelines for Animal Experimentation of Nagasaki University with approval from the Institutional Animal Care and Use Committee. Male ddY mice (5-6 weeks old) were purchased from Japan SLC (Shizuoka, Japan). After shipping, mice were acclimatized to the environment for at least one day before the experiments. Lipopolyplexes were prepared before each experiment. The mice were injected intravenously with complexes at a volume of $200 \mu \mathrm{l}$ per mouse. At $6 \mathrm{~h}$ following the injection, the mice were sacrificed, and the liver, kidney, spleen, heart, and lung were dissected. The activity of luciferase in the tissues was determined as the gene expression.

\subsection{Luciferase assay}

Transfected cells were washed with PBS and then lysed in $200 \mu$ of lysis buffer (pH 7.8 and 0.1 M Tris/HCl buffer containing $0.05 \%$ Triton $\mathrm{X}-100$ and $2 \mathrm{mM}$ EDTA); the lysates were used for luciferase assay. For in vivo experiments, the tissues were homogenized in lysis buffer. The lysis buffer was added in a weight ratio of $3 \mu \mathrm{l} / \mathrm{mg}$ for liver samples, $5 \mu \mathrm{l} / \mathrm{mg}$ for kidney samples and 10 $\mu \mathrm{l} / \mathrm{mg}$ for other organ samples. The homogenates were centrifuged at $21,880 \mathrm{x} g$ for 5 min and the supernatants were used for luciferase assays. Ten microliters of lysate samples were mixed with 50 $\mu \mathrm{l}$ of luciferase assay buffer (Picagene, Toyo Ink, Tokyo, Japan) and the light produced was immediately measured using a luminometer (Lumat LB 9507, EG \& G Berthold, Bad Wildbad, Germany). Luciferase activity was indicated as relative light units (RLU) per mg protein for in vitro experiments and RLU per gram of tissue for in vivo experiments.

\subsection{WST-1 assay}


Cytotoxicity tests of polyplexes and lipopolyplexes on HepG2 cells were carried out using a WST-1 commercially available cell proliferation reagent. The assay is based on cleavage of the tetrazolium salt WST-1 by active mitochondria to produce a soluble colored formazan salt. Since the conversion occurs only with viable cells, it directly correlates with the cell number. The stock solution of WST-1 $(5.5 \mathrm{mM})$ and 1-methoxy PMS $(2 \mathrm{mM})$ was prepared in sterilized PBS and a mixture was prepared by mixing $4.5 \mathrm{ml}$ of WST-1 solution and $0.5 \mathrm{ml}$ of 1-methoxy PMS solution just before the experiments. HepG2 cells were plated at $2.5 \times 10^{4}$ cells/well in 24-well collagen-coated plates (Becton-Dickinson, Franklin Lakes, NL, USA.). The transfection procedure was performed as described above. At $24 \mathrm{~h}$ after transfection, the medium was removed and $1 \mathrm{ml}$ fresh growth medium and $100 \mu \mathrm{l}$ of WST-1 mixture solution were added to each well. The cells were incubated for $2 \mathrm{~h}$ at $37^{\circ} \mathrm{C}$ and absorbance was measured at a wavelength of $450 \mathrm{~nm}$ with a reference wavelength of $630 \mathrm{~nm}$, using a microplate reader (Thermo Fisher Scientific, Inc., USA). The results are shown as a percentage of untreated cells.

\subsection{Statistical Analysis}

The results are reported as the mean with SD of at least three experiments. Statistical analysis was performed using ANOVA and Dunnett's test with Bonferroni's correction for multiple comparison. $P$ $<0.05$ was considered to indicate significance. 


\section{Results}

\subsection{Physicochemical properties of polyplexes and lipopolyplexes}

The particle size and zeta potential of polyplex and lipopolyplex were measured and are shown in Table 1. Polyplex $2 \mathrm{P}$ had $262.7 \mathrm{~nm}$ mean particle size and $23.7 \mathrm{mV}$ mean zeta potential. The addition of DOTMA decreased the particle size and increased the zeta potential. The particle size of lipopolyplex 2P-1D was $114.1 \mathrm{~nm}$, which was 0.4 -fold that of polyplex 2P. Further addition of DOTMA did not change the particle size. The zeta potential of lipopolyplex 2P-1D was $49.1 \mathrm{mV}$, which was 2.1-fold that of polypex 2P. An increase of DOTMA gradually increased the zeta-potential and it reached $57.1 \mathrm{mV}$ with lipopolyplex 2P-4D. Lipopolyplex 2P-2D was observed with transmission electron microscopy (TEM) and the image is shown in Fig. 1. Particles having a diameter of 24-130 nm were observed with low polydispersity on a TEM picture. The liposomal shape of DOTMA was not observed by TEM.

\subsection{Gel retardation assay}

Complex formations of polyplexes and lipopolyplexes were examined by a gel retardation assay (Fig. 2i). Naked pDNA was detected as two bands on agarose gel (A). Polyplex 2P showed two bands of pDNA on the gel (B). A slight amount of naked pDNA was detected for lipopolyplex 2P-1D (C). Bands of pDNA were not detected in lipopolyplex 2P-2D and lipopolyplex 2P-4D (D, E). Polyplex 8P also showed no detection of pDNA bands. When an equal volume of heparin calcium was added to each complex and loaded onto agarose gel, bands of naked pDNA were detected in all complexes (data are not shown).

In order to study the effect of serum on the stability of polyplexes and lipopolyplexes, the complexes were incubated with serum for $15 \mathrm{~min}$ at $37^{\circ} \mathrm{C}$ and thereafter an equal volume of heparin calcium was added to each complex and electrophoresis performed (Fig. 2ii). After serum treatment, 
naked pDNA was detected as bands and fragmentations on the agarose gel (A'). The polyplex 2P showed bands of pDNA and fragmentations on the gel (B'). Bands of pDNA and fragmentations were also detected in lipopolyplex 2P-1D (C'). Bands of pDNA with no fragmentations were detected in lipopolyplex 2P-2D and lipopolyplex 2P-4D (D', E'). Polyplex 8P showed pDNA bands with no fragmentation (F').

\subsection{In vitro transgene efficiency of polyplexes and lipopolyplexes}

In vitro gene expression efficiencies of polyplexes and lipopolyplexes were determined with HepG2 cells in the absence or presence of FBS. Luciferase activity at $24 \mathrm{~h}$ after transfection was measured as the gene expression in the absence of FBS (Fig. 3A). The lipopolyplexes and polyplex $8 \mathrm{P}$ expressed high luciferase activity although polyplex $2 \mathrm{P}$ did not show the gene expression. When transfection was performed in the presence of FBS, the lipopolyplexes and polyplex 8P still expressed high luciferase activity, although polyplex $2 \mathrm{P}$ did not show the gene expression. The commercial transfection reagent, lipofectin, showed $1.65 \times 10^{9} \mathrm{RLU} / \mathrm{mg}$ protein in the absence of FBS and $1.38 \times 10^{9} \mathrm{RLU} / \mathrm{mg}$ protein in the presence of FBS.

Gene expressions mediated by polyplexes and lipopolyplexes were visualized using pEGFP. GFP expression was observed with fluorescent microscopy (Fig. 4). No fluorescence was found in cells treated with naked pDNA (A), polyplex 2P (B), and lipopolyplex 2P-1D (C). Lipopolyplex 2P-2D showed visible GFP expression in several cells (D). Many GFP-positive cells were observed in lipopolyplex 2P-4D (E) and polyplex 8P (F).

\subsection{Intracellular distribution of lipopolyplex}

Intracellular distribution of lipopolyplex 2P-4D was visualized using pEGFP, Rh-PEI, and Hoechist 33342 (Fig. 5i). Fig. 5iA shows a phase contrast picture $24 \mathrm{~h}$ after transfection. Fluorescent pictures are for Hoechst 33342 in Fig. 5iB, for Rh-PEI in Fig. 5iC, and for GFP in Fig. 5iD, 
respectively. The tone of each image was adjusted and overlapped to a merged picture by digital processing (Fig. 5iE). In Fig. 5iE, red dots of Rh-PEI were located mainly in the cytoplasm of most cells but were not observed in nuclei. Among them, strong GFP expression was observed in a few cells. We observed same intracellular distribution of fluorescent-labeled lipid, Rh-DHPE, which is loaded onto the complex (Fig. 5ii).

\subsection{Cytotoxicity of polyplexes and lipopolyplexes}

Cytotoxicity of polyplexes and lipopolyplexes was determined by WST-1 assay (Fig. 6). When cytotoxicity assay was performed in the absence of FBS, the lipopolyplexes and polyplex 8P exhibited significantly lower cell viability compared with untreated cells (Fig. 6A), although polyplex 2P did not show cytotoxicity. In the presence of FBS, all polyplexes and lipopolyplexes showed comparable cell viability to untreated cells (Fig. 6B).

\subsection{In vivo transgene efficiency of polyplexes and lipopolyplexes}

In vivo gene expression efficiencies of polyplexes and lipopolyplexes were examined in ddY male mice. Luciferase activity in several tissues was measured $6 \mathrm{~h}$ after intravenous injection of the complexes (Fig. 7). Polyplex 2P and lipopolyplex 2P-1D showed little luciferase activity in any tissues. High gene expressions were observed in the liver, spleen, and lung with lipopolyplex 2P-2D and lipopolyplex 2P-4D and polyplex 8P. In particular, lipopolyplex 2P-4D showed highest gene expression efficiency. 


\section{Discussion}

PEI is one of the most efficient non-viral vectors and is widely used for in vitro and in vivo gene delivery studies. The transfection efficiency of PEI depends on its molecular weight and structure. In this report, we used branched PEI of molecular weight $25 \mathrm{kDa}$ and compared the transgene efficiency of pDNA/PEI polyplexes to that of pDNA/PEI/DOTMA lipopolyplexes. The transgene efficiency of PEI was also reported to depend on the nitrogen to phosphate (N/P) ratio of pDNA/PEI (Goula et al., 1998; Choosakoonkriang et al., 2003). Polyplex 2P showed little gene expression in in vitro and in vivo experiments (Figs. 3, 4, and 7). Polyplex 2P was found to have large particles with a slightly positive surface charge $(+25 \mathrm{mV})$, as shown in Table 1 . A small amount of PEI may be not enough to compact pDNA. The gel retardation assay indicated that polyplex $2 \mathrm{P}$ was insufficient to bind with pDNA and to protect pDNA from enzymatic degradation in serum. The instability of polyplex 2P should cause low gene expression. On the other hand, a large amount of PEI (polyplex 8P) could form a stable complex with pDNA (Table 1 and Fig. 2) and showed high gene expression in in vitro and in vivo experiments (Figs. 3, 4, and 7). A large amount of PEI, however, was reported to damage the liver and other tissues after its administration to mice (Kawakami et al., 2006; Xiong et al., 2007).

We prepared the lipopolyplex by the addition of cationic lipids, DOTMA, to polyplex 2P. DOTMA is a cationic lipid used as a gene delivery liposome (Felgner et al., 1984; Wollenberg et al., 1999; Hedman et al., 2003). The particle size of the complex was significantly reduced by DOTMA addition and settled to 74-114 nm, indicating pDNA compaction (Table 1). Compacted particles of about $60 \pm 20 \mathrm{~nm}$ were also observed by TEM (Fig. 1). Most lipopolyplex 2P-2D particles had a ball-shaped structure by self-association. In the present study, we added DOTMA ethanol solution to the polyplexes and did not find any liposomal shape by TEM, which was observed in previous reports (Gao et al,. 1996; Lampela et al,. 2003). These results suggest that lipopolyplexes have a compacted core of $\mathrm{pDNA} / \mathrm{PEI}$ and a coating layer of DOTMA. The coating layer may compact the 
core more by ionic bonding, hydrogen bonding, and hydrophobic bonding, and reduce the particle size. pDNA is a large molecule and its compaction is essential for cell internalization. The particle size of complexes is an important factor to control cellular uptake, and small particles should more easily enter cells via an endocytosis pathway than larger ones.

The stability of lipopolyplexes was confirmed by electrophoresis, as shown in Fig. 2. The addition of cationic lipids, DOTMA, to polyplex 2P decreased the dissociation of pDNA from the complex. Lipopolyplex 2P-2D and lipopolyplex 2P-4D completely associated with pDNA (Fig. 2i). The complete association of lipopolyplex 2P-2D and lipopolyplex 2P-4D resulted in protecting pDNA by enzymatic degradation in serum (Fig. 2ii). We also found an increase of zeta potential of polyplex with the addition of DOTMA. The zeta potential of lipopolyplexes reached about $60 \mathrm{mV}$ with lipopolyplex $2 \mathrm{P}-4 \mathrm{D}$, which is similar to the zeta potential of DOTMA, indicating that the surface of lipopolyplexes is covered with DOTMA molecules.

The addition of DOTMA to polyplex 2P remarkably increased the gene expression in HepG2 cells in the absence or presence of FBS. Especially in the presence of FBS, lipopolyplex 2P-2D and 2P-4D had a high gene expression, comparable with polyplex $8 \mathrm{P}$ and a commercial transfection reagent, lipofectin (Fig. 3B). It is worth noting that FBS did not depress the gene expression of these lipopolyplexes. Lipopolyplexes of PEI with DOTAP were also reported to show high transgene efficiencies, even in the presence of FBS (Garcia et al., 2007). The high gene expression of lipopolyplexes in the presence of FBS indicates their usefulness under in vivo conditions. Low gene expression of lipopolyplex 2P-1D can be explained by the instability of the complex in serum, as shown in the results of electrophoresis (Fig. 2ii). These gene expression results were confirmed by the cell numbers visualized by pEGFP (Fig. 4). Lipopolyplex 2P-2D, lipopolyplex 2P-4D, and polyplex 8P showed large numbers of gene-expressing cells. Furthermore, we observed the intracellular distribution of lipopolyplexes 2P-4D labeled with Rh-PEI or Rh-DHPE in the presence of FBS. The red dots of Rh-PEI were located mainly in the cytoplasm of most cells but were not 
observed in nuclei, suggesting that the complexes were conveyed into late endosomes, and finally accumulated in lysosomes (Fig. 5i). We observed the same intracellular distribution of fluorescent-labeled lipid, Rh-DHPE, which is loaded into the complex (Fig. 5ii), indicating that lipopolyplexes were taken up in the cells by the endocytotic pathway, and both PEI and lipids are responsible for the delivery of pDNA into cells. Lipopolyplex must be able to enhance internalization in many cells. Although many cells showed red dots of Rh-PEI in the cytoplasm, only a few cells strongly expressed GFP. These results may indicate the importance of pDNA release from endosome and pDNA delivery into the nucleus for successful gene expression. It was reported that only a few pDNA reached the nuclei, although a large amount of pDNA complexes were localized in the cytoplasm after cellular internalization (Lechardeur et al, 2005; Hama et al, 2005). The high gene expression of lipopolyplexes may have been caused by both membrane fusion characteristics of DOTMA molecules and the proton-sponge effect of PEI (Chen et al, 2007; Hattori et al, 2007).

The cytotoxicity of lipopolyplexes was measured by the WST-1 assay in HepG2 cells. Lipopolyplexes showed little cytotoxicity in the presence of FBS (Fig. 6B), although the lipopolyplexes and polyplex 8P had significant cytotoxicity in the absence of FBS (Fig. 6A). The serum-starving condition may influence cell sensitivity to highly cationic complexes. The cytotoxicity of lipopolyplex 2P-4D must cause its low gene expression in the absence of FBS (Fig. 3A); nevertheless, gene delivery in the presence of FBS is important for clinical use.

Based on these results, we tested the efficiency of lipopolyplexes for in vivo gene delivery. After intravenous injection of lipopolyplexes into mice, luciferace activity in several tissues was determined by luminescent analysis. Gene expression was slight in all organs with polyplex $2 \mathrm{P}$ and lipopolyplex 2P-1D. Instability of these complexes in serum might be the reason for their insufficient gene expression. In contrast, high gene expression in the liver, spleen, and lung was observed in lipopolyplex 2P-2D, lipopolyplex 2P-4D, and polyplex 8P. In particular, lipopolyplex 2P-4D showed the highest gene expression among them. The high gene expression of lipopolyplexes 
may be reflected by the stability of the complex in the presence of FBS, membrane fusion characteristics of DOTMA molecules, and the proton-sponge effect of PEI.

Thus, these results confirm the usefulness of PEI lipopolyplex with DOTMA and pDNA in in vitro and in vivo gene delivery. Further experiments may be necessary to use them clinically. 


\section{Acknowledgements}

We would like to thank Dr. Kawakami and Dr. Niidome for their assistance with the Zetasizernano measurement. We would also like to thank Dr. Suematsu, from the core electron microscopy facility of Nagasaki University, for his technical assistance with TEM. 


\section{References}

Boussif, O., Lezoualc'h, F., Zanta, M.A., Mergny, M.D., Scherman, D., Demeneix, B., Behr, J.P., 1995. A versatile vector for gene and oligonucleotide transfer into cells in culture and in vivo: polyethylenimine. Proc. Natl. Acad. Sci. U S A., 92, 7297-7301.

Chen, J.L., Wang, H., Gao, J.Q., Chen, H.L., Liang, W.Q., 2007. Liposomes modified with polycation, characterization and transfection in vitro. Int. J. Pharm., 343, 255-261.

Choosakoonkriang, S., Lobo, B.A., Koe, G.S., Koe, J.G., Middaugh, C.R., 2003. Biophysical characterization of PEI/DNA complexes. J. Pharm. Sci., 92, 1710-1722.

Edelstein, M.L., Abedi, M.R., Wixon, J., 2007. Gene therapy clinical trials worldwide to 2007-an update. J. Gene Med., 9, 833-842.

Fillion, M.C., Phillips, N.C., 1998. Major limitations in the use of cationic liposomes for DNA delivery. Int. J. Pharm., 162, 159-170.

Fischer, D., Bieber, T., Li, Y., Elsässer, H.P., Kissel, T., 1999. A novel non-viral vector for DNA delivery based on low molecular weight, branched polyethylenimine: effect of molecular weight on transfection efficiency and cytotoxicity. Pharm. Res., 16, 1273-1279.

Gao, X., Huang, L., 1995. Cationic liposome-mediated gene transfer. Gene. Ther., 2, 710-722.

Gao, X., Huang, L., 1996. Potentiation of cationic liposome-mediated gene delivery by polycations. Biochemistry, 35, 1027-1036.

García, L., Buñuales, M., Düzgüneş, N., Tros de Ilarduya, C., 2007. Serum-resistant lipopolyplexes for gene delivery to liver tumour cells. Eur. J. Pharm. Biopharm., 67, 58-66.

Godbey, W.T., Wu, K.K., Mikos, A.G., 1999. Poly(ethylenimine) and its role in gene delivery. J. Control. Release, 60, 149-160.

Goldman, C.K., Soroceanu, L., Smith, N., Gillespie, G.Y., Shaw, W., Burgess, S., Bilbao, G., Curiel, D.T., 1997. In vitro and in vivo gene delivery mediated by a synthetic polycationic amino polymer. 
Nat. Biotechnol., 15, 462-466.

Goula, D., Benoist, C., Mantero, S., Merlo, G., Levi, G., Demeneix, B.A., 1998. Polyethylenimine-based intravenous delivery of transgenes to mouse lung. Gene Ther., 5, 1291-1295.

Hama, S., Akita, H., Ito, R., Mizuguchi, H., Hayakawa, T., Harashima, H., 2006. Quantitative comparison and nuclear transcription between adenoviral and lipoplex systems. Mol. Ther., 13, 786-794.

Hattori, Y. and Maitani, Y., 2007. Low-molecular-weight polyethylenimine enhanced gene transfer by cationic cholesterol-based nanoparticle vector. Biol. Pharm. Bull., 30, 1773-1778.

Hedman, M., Hartikainen, J., Syvänne, M., Stjernvall, J., Hedman, A., Kivelä, A., Vanninen, E., Mussalo, H., Kauppila, E., Simula, S., Närvänen, O., Rantala, A., Peuhkurinen, K., Nieminen, M.S., Laakso, M., Ylä-Herttuala, S., 2003. Safety and feasibility of catheter-based local intracoronary vascular endothelial growth factor gene transfer in the prevention of postangioplasty and in-stent restenosis and in the treatment of chronic myocardial ischemia: phase II results of the Kuopio Angiogenesis Trial (KAT). Circulation, 107, 2635-2637.

Itaka, K., Harada, A., Yamasaki, Y., Nakamura, K., Kawaguchi, H., Kataoka, K., 2004. In situ single cell observation by fluorescence resonance energy transfer reveals fast intra-cytoplasmic delivery and easy release of plasmid DNA complexed with linear polyethylenimine. J. Gene Med., 6, 76-84.

Kawakami, S., Ito, Y., Charoensit, P., Yamashita, F., Hashida, M., 2006. Evaluation of proinflammatory cytokine production induced by linear and branched polyethylenimine/plasmid DNA complexes in mice. J. Pharmacol. Exp. Ther., 317, 1382-1390.

Kichler, A., Leborgne, C., Coeytaux, E., Danos, O., 2001. Polyethylenimine-mediated gene delivery: a mechanistic study. J. Gene Med., 3, 135-144.

Kircheis, R., Wightman, L., Wagner, E., 2001. Design and gene delivery activity of modified 
polyethylenimines. Adv. Drug Deliv. Rev., 53, 341-358.

Lampela, P., Elomaa, M., Ruponen, M., Urtti, A., Männistö, P.T., Raasmaja, A., 2003. Different synergistic roles of small polyethylenimine and Dosper in gene delivery. J. Control. Release, 88, 173-183.

Lechardeur, D., Verkman, A.S., Lukacs, G.L., 2005. Intracellular routing of plasmid DNA durring non-viral gene transfer. Adv. Drug Deliv. Rev., 57, 755-767.

Lee, C.H., Ni, Y.H., Chen, C.C., Chou, C., Chang, F.H., 2003. Synergistic effect of polyethylenimine and cationic liposomes in nucleic acid delivery to human cancer cells. Biochim. Biophys. Acta., $1611,55-62$.

Li, S., Huang, L., 1997. In vivo gene transfer via intravenous administration of cationic lipid-protamine-DNA (LPD) complexes. Gene Ther., 4, 891-900.

Pelisek, J., Gaedtke, L., DeRouchey, J., Walker, G.F., Nikol, S., Wagner, E., 2006. Optimized lipoplex formulations for gene transfer to human colon carcinoma cells under in vitro conditions. $\mathrm{J}$ Gene Med., 8, 186-197.

Pollard, H., Remy, J.S., Loussouarn, G., Demolombe, S., Behr, J.P., Escande, D., 1998. Polyethylenimine but not cationic lipids promotes transgene delivery to the nucleus in mammalian cells. J. Biol. Chem., 273, 7507-7511.

Sorgi, F.L., Bhattacharya, S., Huang, L., 1997. Protamine sulfate enhances lipid-mediated gene transfer. Gene. Ther., 4, 961-968.

Tang, M.X., Szoka, F.C., 1997. The influence of polymer structure on the interactions of cationic polymers with DNA and morphology of the resulting complexes. Gene Ther., 4, 823-832.

Verma, I.M., Somia, N., 1997. Gene therapy -- promises, problems and prospects. Nature, 389, 239-242.

Wollenberg, B., Kastenbauer, Mundl, H., Schaumberg, J., Mayer, A., Andratschke, M., Lang, S., Pauli, C., Zeidler, R., Ihrler, S., Löhrs, Naujoks, K., Rollston, R., 1999. Gene therapy--phase I trial 
for primary untreated head and neck squamous cell cancer (HNSCC) UICC stage II-IV with a single intratumoral injection of hIL-2 plasmids formulated in DOTMA/Chol. Hum. Gene. Ther., $10,141-147$.

Xiong, M.P., Laird Forrest, M., Ton, G., Zhao, A., Davies, N.M., Kwon, G.S., 2007. Poly(aspartate-g-PEI800), a polyethylenimine analogue of low toxicity and high transfection efficiency for gene delivery. Biomaterials, 28, 4889-4900. 


\section{Table}

Table 1. The particle size and zeta potential of polyplexes and lipopolyplexes

\begin{tabular}{ccc}
\hline Complex & Particle size $(\mathrm{nm})$ & Zeta potential $(\mathrm{mV})$ \\
\hline Polyplex 2P & $262.7 \pm 86.0$ & $23.7 \pm 0.6$ \\
Lipopolyplex 2P-1D & $114.1 \pm 21.5$ & $49.1 \pm 0.6$ \\
Lipopolyplex 2P-2D & $73.9 \pm 26.8$ & $54.8 \pm 0.8$ \\
Lipopolyplex 2P-4D & $110.5 \pm 28.8$ & $57.1 \pm 1.3$ \\
Polyplex 8P & $24.8 \pm 1.7$ & $40.8 \pm 2.5$ \\
\hline
\end{tabular}

Each value is the mean $\pm \operatorname{SD}(n=3)$. 
Figure Legends

Fig. 1

Transmission electron microscopy (TEM) image of lipopolyplex 2P-2D. Bar represents $200 \mathrm{~nm}$. Magnification x 25000

Fig. 2

Gel retardation assay (i) and serum protection assay (ii) was performed with electrophoresis for polyplexes 2P (B), lipopolypxes 2P-1D (C), 2P-2D (D), 2P-4D (E), and polyplex 8P (F). Naked pDNA (A) was used as a control. For the serum stability assay, complexes were incubated with fresh mouse serum for $15 \mathrm{~min}$ at $37^{\circ} \mathrm{C}$ and loaded onto an agarose gel with an equal volume of heparin calcium to separate pDNA from the complex (A'-F').

Fig. 3

Transfection efficiencies of polyplexes and lipopolyplexes in the absence (A) or presence (B) of FBS. HepG2 cells were incubated for $2 \mathrm{~h}$ with pCMV-Luc complexed with PEI and DOTMA at various ratios. Twenty-four hours after transfection, cells were lysed for quantification of luciferase activity. The values are the means of luciferase activity (RLU/ mg protein) with $\mathrm{SD}(\mathrm{n}=3) . * * p<0.001 \mathrm{vs}$ polyplex 8P, \#\# $p<0.001$.

Fig. 4

Fluorescent microscopy images of HepG2 cells transfected with pEGFP/PEI/DOTMA complexes. HepG2 cells were treated with naked pDNA (A), polyplex 2P (B) and lipopolyplexes 2P-1D (C), 2P-2D (D), 2P-4D (E) and polyplex 8P (F) for $2 \mathrm{~h}$ and the expression of GFP was monitored at $24 \mathrm{~h}$. Magnification x 10 
Fig. 5

Cell viability of HepG2 cells treated with lipopolyplexes was measured by WST-1 assay. Cells were incubated with various ratios of polyplexes and lipopolyplexes in the absence (A) or presence (B) of FBS for $2 \mathrm{~h}$ and cell viability was measured at $24 \mathrm{~h}$ after treatment. Data represent the percentage to untreated cells. Each value is the mean with $\mathrm{SD}(\mathrm{n}=3) .{ }^{* *} p<0.001$ vs control, $\# p<0.05$, \#\# $p<$ 0.001 .

Fig. 6

Intracellular distribution of lipopolyplex 2P-4D labeled with fluorescent dyes including rhodamin-PEI (Rh-PEI, Fig. 6i) and lissamine rhodamine B 1,2-dihexadecanoylsn-glycero-3-phosphoethanolamine, triethylammonium salt (Rh-DHPE, Fig. 6ii). HepG2 cells were treated with lipopolyplexes for $2 \mathrm{~h}$. Intracellular distribution of complexes and GFP expression (green) was observed 24 hours after transfection. Nuclei were stained by Hoechst 33342 (blue). Phase contrast image (A), nuclei staining (B), distribution of Rh-PEI dots or Rh-DHPE dots (C), expression of GFP (D) and merged image (E) are indicated. Magnification x 200

Fig. 7

In vivo transgene efficiencies of polyplexes and lipopolyplexes, expressed as RLU per gram tissue. The complexes were injected intravenously in mice ( $40 \mu \mathrm{g}$ DNA per mouse). At $6 \mathrm{~h}$ after administration, mice were sacrificed and each organ was dissected for quantification of luciferase activity. Each bar is the mean with $\mathrm{SD}(\mathrm{n}=3-6) .{ }^{*} p<0.05$ vs polyplex $8 \mathrm{P}, \# p<0.05$, \#\# $p<0.001$. 
Figures

Fig. 1

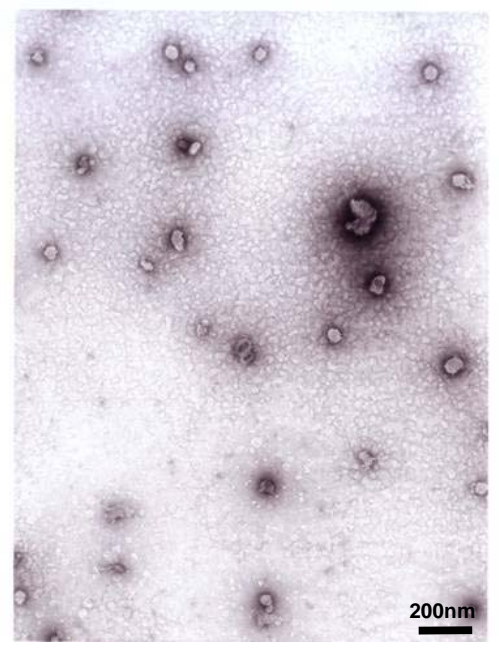


Fig. 2

(i)

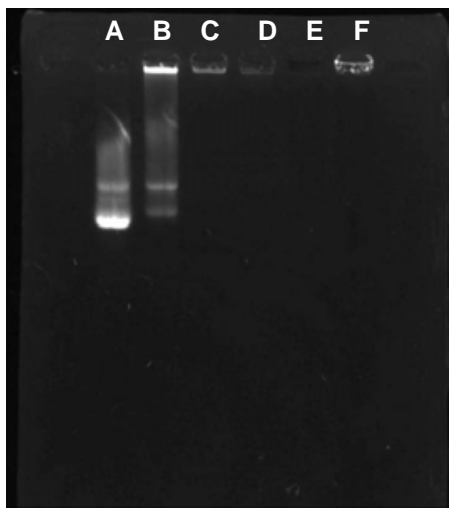

(ii)

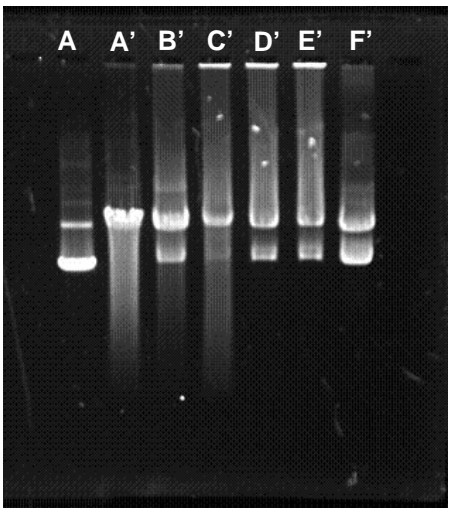


Fig. 3

(A)

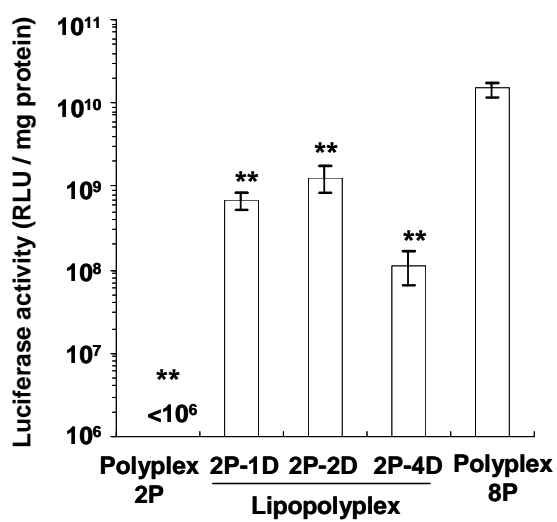

(B)

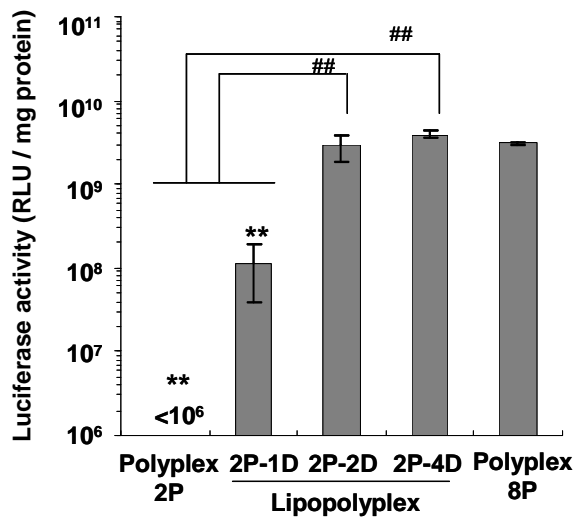


Fig. 4

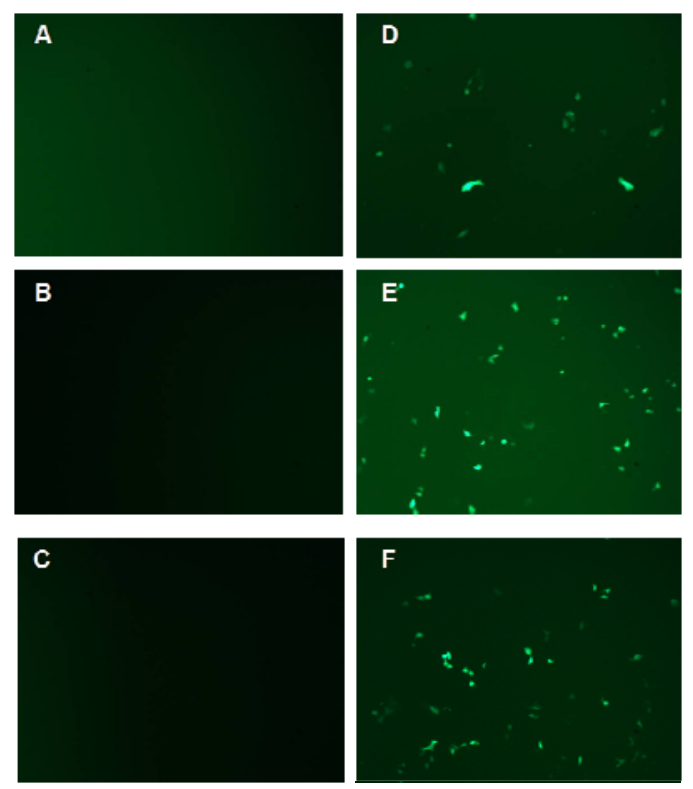


Fig. 5

(o)
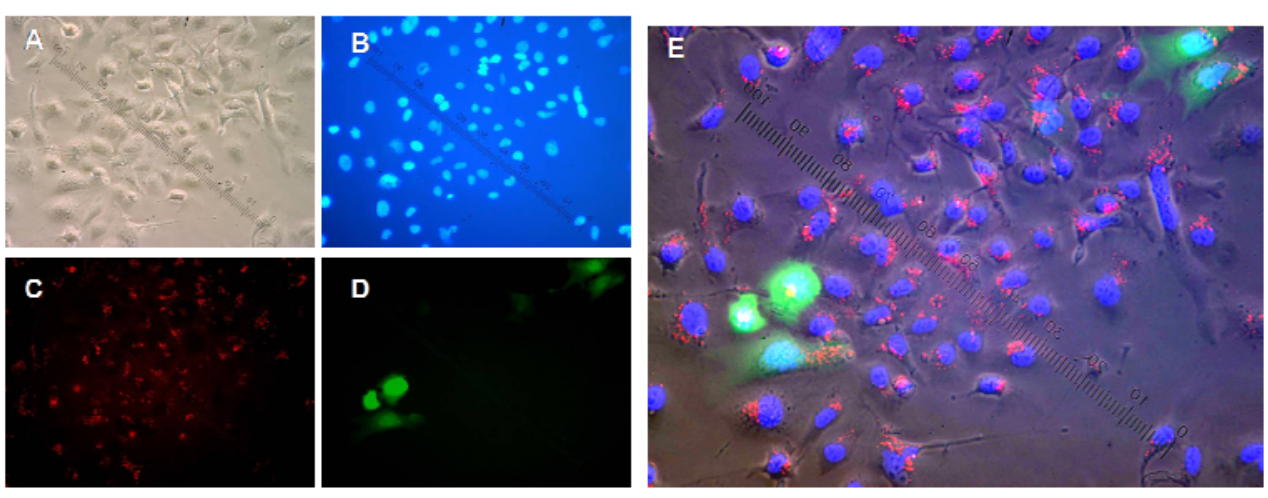

(II)
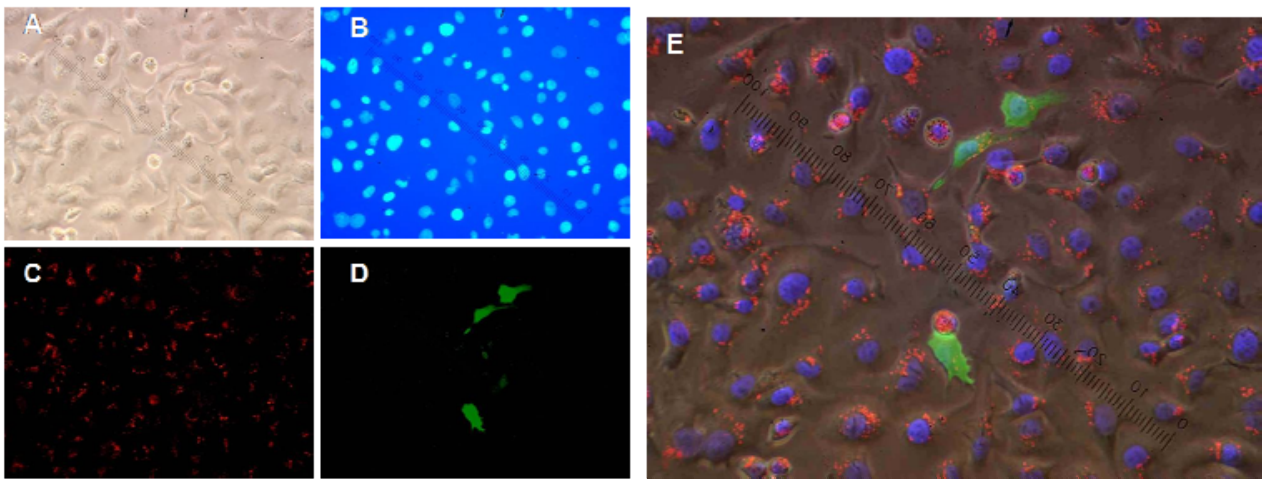
Fig. 6

(A)

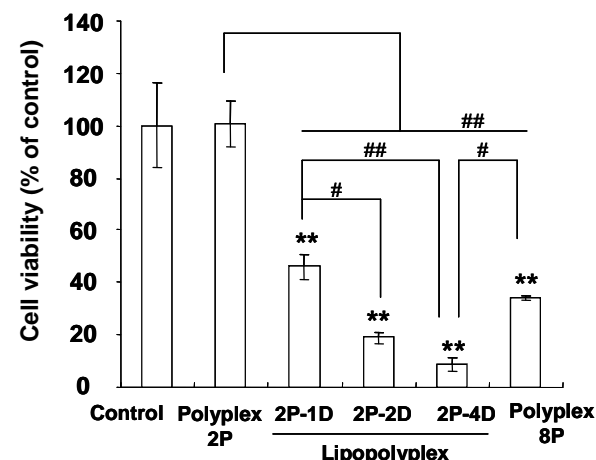

(B)

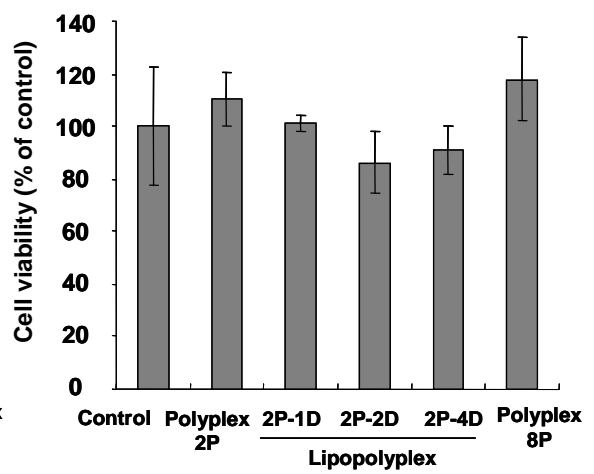


Fig. 7

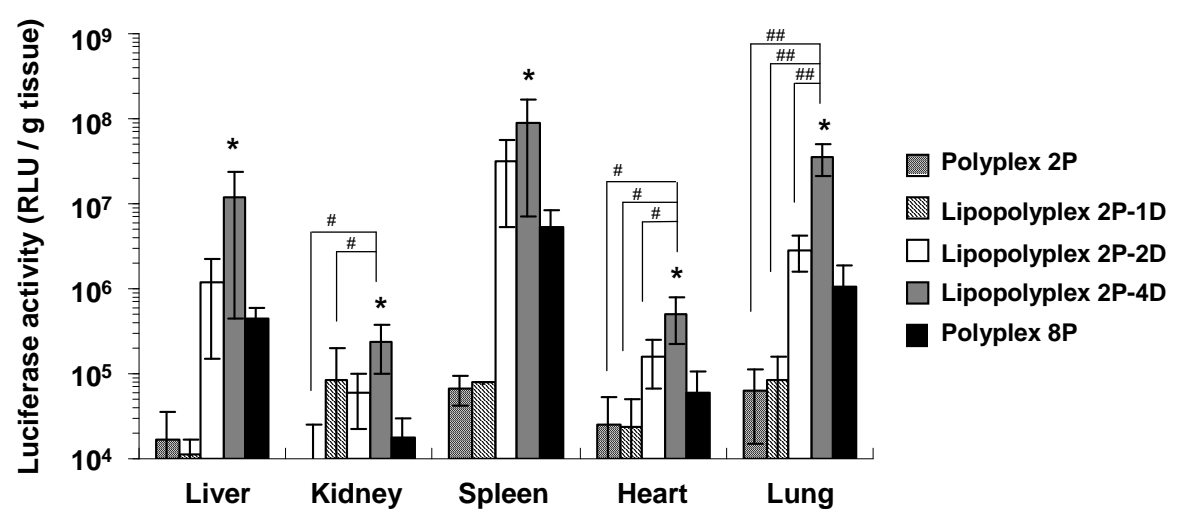

